\title{
Characterization of the channel diffusion in a sodium tetrasilicate glass via molecular-dynamics simulations
}

\author{
Emmanuel Sunyer, Philippe Jund* and Rémi Jullien \\ Laboratoire des Verres, Université Montpellier 2, Place E. Bataillon, Case 069, \\ 34095 Montpellier, France \\ * Laboratoire de Physicochimie de la Matière Condensée, CNRS-Université Montpellier 2, \\ Place E. Bataillon Case 03, 34095 Montpellier, France
}

\begin{abstract}
We study the structural and dynamical characteristics of the sodium atoms inside and outside the "diffusion channels" in glassy $\mathrm{Na}_{2} \mathrm{O}-4 \mathrm{SiO}_{2}$ (NS4) using classical molecular dynamics. We show that on average neither energetic arguments nor local environment considerations can explain the increased density of sodium atoms inside the subspace made of the channels. Nevertheless we show that at low temperature the mean square displacement of the sodium atoms inside this subspace is significantly larger than the one of the atoms outside the channels.
\end{abstract}

PACS numbers: 61.20.Ja, 61.43.Fs, 66.30.Hs

The diffusion of alkali atoms in silicate glasses is an important matter under investigation since several years. In particular the properties of sodium atoms inside the amorphous tetrahedral network of silica have been the topic of both experimental work [1] and moleculardynamics simulations (MD) [2] since a simple glass like $\mathrm{Na}_{2} \mathrm{O}-4 \mathrm{SiO}_{2}$ (NS4) can be used as a prototype for more complicated glasses. The question of how the alkali atoms diffuse inside the glassy network is still a matter of debate and a generally accepted theory of ion transport in glasses is still missing [3., In. In a previous MD study of NS4 we have shown that the ions follow preferential pathways ("channels") inside the glassy matrix [5]. Nevertheless contrarily to the popular idea proposed by Greaves [6] and developed in further studies [7], these channels are neither static nor due to a microsegregation of the sodium atoms but have to be seen dynamically in the sense that the channels are those regions of space in which a great number of sodiums have passed during a given simulation time. The existence of these channels gives rise to a pre-peak in the structure factor at around $q=0.95 \AA^{-1}$ seen both in experiments [8] and classical MD simulations [9]. In this last paper Horbach et al. show that the slow dynamics of the sodium atoms is closely related to the one of the underlying silica matrix which is coherent with the fact that there exists a strong correlation between the channels and the location of the non-bridging oxygen atoms, as shown in previous MD studies [10,11].

Once the existence of the channels is established, the next step is naturally to analyze their characteristics and to determine why the sodium atoms take these preferential pathways. This is the aim of the present study. In that direction we analyze the potential energy and the local structure of the sodium atoms whether they are INside the channels $\left(\mathrm{Na}_{\mathrm{In}}\right)$ or OUTside the channels (NaOut). It is indeed generally believed that the diffusion of the ions occurs via "hopping motions between well-defined potential minima" 淊 which we should be able to detect in our simulations. We analyze also the time evolution of the sodium densities inside and outside the channels which permits us to detect the dynamics of the channels as a function of temperature and to quantify the differences between the sodium densities, differences that so far have only been suggested [9]. To elucidate the diffusion mechanisms of the $\mathrm{Na}$ atoms we determine a characteristic "residence" time inside the channels and we study the mean square displacements versus temperature of the $\mathrm{Na}_{\mathrm{In}}$ and $\mathrm{Na}$ Out atoms.

Our system is made of 648 particles, namely $N_{\mathrm{Na}}=86$ sodium, 173 silicon and 389 oxygen atoms, confined in a cubic simulation box of edge length $L=20.88 \AA$ to which we apply periodic boundary conditions. The density is thus the experimental one, i.e. $2.38 \mathrm{~g} \mathrm{~cm}^{-3}$ [12]. The interaction potential used in our simulations is a modified version of the one proposed by Kramer et al 13. and its complete description can be found in 11. It has been shown in previous studies that this potential is able to describe reliably many structural and dynamical properties of different sodium silicate melts [9,14] and especially NS4 [5, 11]. We have used as initial structure a $\beta$-cristobalite crystal in which we have randomly substituted the appropriate number of $\mathrm{SiO}_{4}$ tetrahedra by $\mathrm{Na}_{2} \mathrm{O}_{3}$ "molecules". Subsequently the system is melted and equilibrated in the liquid phase at high temperature, i.e. $4000 \mathrm{~K}$, for 50000 time steps ( $\equiv 35 \mathrm{ps}$ ). Then it is cooled down very rapidly with a linear cooling schedule at a quench rate of $2.3 \times 10^{14} \mathrm{~K} \mathrm{~s}^{-1}$. During the quenching process, the configurations of the system (positions and velocities of all the atoms) are saved at different temperatures $(T \approx 4000,3100,2500,2300,1900$ and $1700 \mathrm{~K}$ ). These configurations are subsequently used as starting points of production runs of 2 million steps ( $\equiv 2.8 \mathrm{~ns})$ performed in the micro-canonical ensemble $[(N, V, E)=$ const $]$. During these production runs we save 2000 configurations equally spaced in time. The glass transition temperature of the system can be roughly estimated from the location of the bend in a plot of the 
potential energy vs $T$ to be around $T_{g} \approx 2400 \mathrm{~K}$. At each temperature the results have been averaged over three independent samples.

As mentioned above, we have shown in previous studies [5, 11] that at low temperature the sodium atoms diffuse through the (quasi-)frozen silica matrix within a subset of the total available space. This subset is made of pockets (typical size 3-6 $\AA$ ) connected together via small pathways (similar in a sense to a neuronal network) and this network is what we call channels. Our aim in this work is to characterize the channel diffusion of the sodium atoms, i.e. to study the possible structural and dynamical differences between the $\mathrm{Na}_{\mathrm{In}}$ and the $\mathrm{NaOut}$ atoms. In order to determine the channels, we divide the simulation box in $N_{t o t}=20^{3}$ small distinct cubes of volume $\approx 1 \AA^{3}$ and determine the number density of the $\mathrm{Na}$ atoms in each of these cubes during the $2.8 \mathrm{~ns}$ of the simulation. Then we consider only the upper $10 \%$ of all the visited cubes. These cubes form "the core" of the channels (the space in which the sodium atoms have been the more often) or shortly "the channels". More details can be found in Ref. [5, 11]. Once the channels have been defined via this time integration, we can go back and determine for every time $t, \mathrm{Na}_{\mathrm{In}}$ and $\mathrm{Na}_{\mathrm{O} u t}$ and start our investigations.

Why do the Na atoms visit a specific fraction of the total available space more frequently at low temperature? The first explanation that comes to mind is that the visited sites are more favorable energetically. To check this idea, we have calculated the individual potential energy distributions of the sodium atoms at $1700 \mathrm{~K}$ whether they are inside or outside the channels. The two distributions have been averaged over the 2000 configurations saved at $1700 \mathrm{~K}$ and over the 3 independent samples and are represented in FIG. 1. It is obvious in FIG. 1 that there is no significant difference between the distribution of $\mathrm{Na}_{\text {In }}$ and $\mathrm{Na}_{\mathrm{Out}}$ : both are gaussians centered approximately at $-3.8 \mathrm{eV}$ with a Half Width at Half Maximum of $\approx 0.6 \mathrm{eV}$. It appears therefore that the energy is not the driving force explaining why the $\mathrm{Na}$ atoms are inside the channels. One may argue that the time average can smear out the effect but in our previous study we have shown that the sodium atoms visit sites previously occupied by other sodium atoms [5] and therefore if these sites were favorable energetically it should appear in FIG. 1. We have also calculated the energy distributions of Si and $\mathrm{O}$ atoms (not shown in FIG. 1 ) and found that they have an average energy of $-35 \mathrm{eV}$ and $-12 \mathrm{eV}$ respectively. In fact, the individual potential energy distributions reflect the whole environment of the Na atoms. Therefore even if there exists a structural difference at short range, it will not be visible in Fig. 1 because of all the other (long-range) interactions. It is hence justified to look more precisely at the environment of the sodium atoms via the radial pair distribution function and the integrated number of neighbors. For a given $\alpha-\beta$ pair they are defined by:

$$
g(r)_{\alpha-\beta}=\frac{V}{4 \pi r^{2} N_{\alpha} d r} d n_{\beta}
$$

and,

$$
N(r)_{\alpha-\beta}=\frac{N_{\alpha}}{V} \int_{0}^{r} 4 \pi r^{2} g(r)_{\alpha-\beta} d r
$$

Note that the number of first nearest $\beta$ neighbors around species $\alpha$, is given by the value of $N(r)_{\alpha-\beta}$ at the first minimum of $g_{\alpha-\beta}(r)$. In FIG. 2 we have represented $g(r)$ and $N(r)$ at $T=1700 \mathrm{~K}$ for (a) $\mathrm{Na}_{\mathrm{Out}}-\mathrm{Na}, \mathrm{Na}_{\mathrm{In}}-\mathrm{Na}$ and $\mathrm{Na}-\mathrm{Na}$ pairs, (b) $\mathrm{Na}_{\mathrm{Out}}-\mathrm{O}, \mathrm{Na}_{\mathrm{In}}-\mathrm{O}$ and $\mathrm{Na}-\mathrm{O}$ pairs and (c) $\mathrm{Na}_{\mathrm{Out}}-\mathrm{Si}, \mathrm{Na}_{\mathrm{In}}-\mathrm{Si}$ and $\mathrm{Na}-\mathrm{Si}$ pairs. The major differences can be seen in (a) where it appears that a $\mathrm{Na}_{\text {In }}$ has on average 1 supplemental $\mathrm{Na}$ neighbor compared to a NaOut atom. This goes together with a reduction of the number of nearest $\mathrm{Si}$ neighbors (c). On the contrary the local oxygen environment (b) seems to be the same for the sodiums inside and outside the channels. Finally concerning the nearest neighbor distances with the other species there is no significant change between $\mathrm{Na}_{\mathrm{In}}$ and NaOut except a slight decrease $(0.1 \AA)$ of the $\mathrm{Na}_{\mathrm{In}}-\mathrm{Na}$ distance compared to the distance between $\mathrm{Na}_{\mathrm{Out}}$ and the other sodium atoms. It appears therefore that the local structure of the sodium atoms inside and outside the channels is not significantly different in order to explain the existence of the channels. Nevertheless it appears from the study of the radial pair distribution functions that on average the $\mathrm{Na}_{\mathrm{In}}$ atoms have more $\mathrm{Na}$ neighbors than the NaOut atoms. Therefore it is justified to calculate at any instant $t$ the number of sodiums inside the channels $\left(N_{\mathrm{Na}}^{\mathrm{In}}(t)\right)$ and the number of sodiums outside the channels $\left(N_{\mathrm{Na}}^{\mathrm{Out}}(t)\right)$. These quantities are shown in FIG. 3 at $1700 \mathrm{~K}(\mathrm{a}), 2300 \mathrm{~K}$ (b) and $3100 \mathrm{~K}$ (c). A first information can be obtained from the shape of the curves at the different temperatures. At $1700 \mathrm{~K}$ (a), the fact that the curves become flat after $\approx 0.5 \mathrm{~ns}$ indicates that the channels are well defined through the frozen silica matrix and are made almost of the same small cubes during the rest of the simulation. At $2300 \mathrm{~K}$ (b), the situation is rather different since $N_{\mathrm{Na}}^{\mathrm{In}}$ goes through a maximum (and consequently $N_{\mathrm{Na}}^{\mathrm{Out}}$ goes through a minimum) after $\approx 1.4 \mathrm{~ns}$. This is a signature of the residual motion of the silica matrix which is only quasi-frozen and hence a manifestation of the motion of the channels. In fact, the channels that we have defined in our calculation at that temperature are the common part of the "moving" channels we could have defined over consecutive fractions of the whole simulation. It is therefore reasonable to assume that the real channels at $\approx 1.4$ ns (half the simulation length) are closest to the channels that we have defined over the whole simulation. At $3100 \mathrm{~K}$ (c), the fact that the curves are flat indicates that the channels do not really exist anymore. What we have defined as channels at that temperature are only randomly distributed 
clusters of density fluctuations. Actually, the matrix is melted and the sodium atoms may visit the entire accessible space (the system is ergodic) and hence the location of the clusters is completely random and depends crucially on the length of the run. Thus, the definition of the channels is quite artificial at high temperature. Nevertheless when we compare the values of $N_{\mathrm{Na}}^{\mathrm{In}}$ after $\approx 1.4 \mathrm{~ns}$ for the different temperatures we do not see an increase of $N_{\mathrm{Na}}^{\mathrm{In}}$ with increasing temperature contrarily to what was suggested by Meyer et al. [8] to explain an increase of the quasielastic amplitude in the dynamic structure factor of sodium disilicate [NS2] melts. Finally we see in FIG. 3 that for all the temperatures the major part of the sodium atoms is outside the channels. Nevertheless as shown in [5] the total volume of the channels is small $(\approx 10 \%$ at $2000 \mathrm{~K})$ therefore the sodium densities can be very different inside and outside the channels. In FIG. 4 we have represented the sodium density $\rho_{\mathrm{Na}}$ inside and outside the channels (which is simply $N_{\mathrm{Na}}^{\mathrm{In}} / V_{\text {chan }}$ and $N_{\mathrm{Na}}^{\mathrm{Out}} /\left(L^{3}-V_{\text {chan }}\right)$ where $V_{\text {chan }}$ is the volume of the channels) as a function of $T$. It is immediately noticeable that the sodium density inside the channels is higher than the one outside and in particular at low temperature (below $T_{g}$ ). So far we knew that the channels are a subspace highly visited by the sodium atoms (they have been defined this way) but now we can conclude in addition that they are also a subspace of high sodium density. We emphasize that a higher sodium concentration does not imply necessarily a clustering of alkalis [6,7]. Actually we have shown that no clustering can be observed on a single snapshot of the simulation box [5]. It is also noticeable that the two curves converge towards the limit of uniform density at high temperature. This is another evidence that at high temperature the channels do not exist and that what we have defined as channels are randomly distributed clusters.

Once we know the position of the sodium atoms with respect to the channels it is naturally of interest to calculate a mean "residence" time of the $\mathrm{Na}$ atoms within the channels. This can be done by determining the probability $P(0, t)$ of a sodium atom inside the channels at $t=0$ to be inside the channels after a time $t . P(0, t)$ is given by:

$$
P(0, t)=\frac{1}{N_{\mathrm{Na}}^{\mathrm{In}}(0)} \sum_{i=1}^{N_{\mathrm{Na}}^{\mathrm{In}}(0)} n_{i}(t)
$$

with:

$$
\left\{\begin{array}{l}
n_{i}=0 \text { outside the channels } \\
n_{i}=1 \text { inside the channels }
\end{array}\right.
$$

This probability is represented in FIG. 5 at different temperatures. Since we save the configurations every $1.4 \mathrm{ps}$ (1000 MD steps), the fast decay from 1 at shorter times can not be seen in Fig. 5 but since the channels have been determined with this "time step" we have decided to calculate $P(0, t)$ in the same conditions. This means therefore that our probability is slightly overestimated since we do not take into account the vibrational effects. In Fig. 5 we see that $P(0, t)$ decreases and converges towards the long time limit $N_{\mathrm{Na}}^{\mathrm{In}} / N_{\mathrm{Na}}$ very rapidly at high temperature while at low temperature $(T \leq 2300 \mathrm{~K})$ this limit is not reached after $1.5 \mathrm{~ns}$. After subtracting the long time limit, these curves can be fitted reasonably well by an exponential function with a time constant $\tau$ which is represented versus $1 / \mathrm{T}$ in a linear-log plot in the inset of FIG. 5. From this inset it is clear that $\tau(T)$, the typical residence time, shows an Arrhenius behavior with an activation energy around $1.45 \mathrm{eV}$. This activation energy is larger than the activation energy of the $\mathrm{Na}$ diffusion process and other more specific characteristics of the Na displacement (hops between preferential sites, forward-backward jumps, decorrelation mechanism) which is around $1.3 \mathrm{eV}$ [5]. This seems to indicate that the mechanism governing the expulsion of the $\mathrm{Na}$ ions outside the channels is energetically more costly than the other mechanisms controlling the mean displacement of the ions.

Finally we have decided to investigate the influence of the location of the sodium atoms on their diffusion by calculating "conditional" mean squared displacements (MSD) $\left(R^{2}(t)=\left\langle|\vec{r}(t)-\vec{r}(0)|^{2}\right\rangle\right)$. On the one hand we have calculated $d^{2}(t)_{\text {In/Out }}$ which is the "MSD" of the atoms inside/outside the channels at time $t=0$ and $t \neq 0$ (this is a reasonable calculation since we know that $P(0, t)$ remains non zero over the length of the simulation). These quantities are represented in Fig. 6 for (a) 1700 K, (b) 1900 K and (c) $2300 \mathrm{~K}$. On the other hand we have calculated $R^{2}(t)_{\text {In/Out }}$ strictly speaking: in that case the atoms are continuously inside/outside the channels between 0 and $t$. In this latter case the number of steps an $\mathrm{Na}$ atom is consecutively inside/outside the channels is small especially at high temperature therefore this quantity is only represented for $1700 \mathrm{~K}$ in the inset of FIG. 6 (a). From the results represented in FIG. 6 we see that at low temperature $(T=1700,1900 \mathrm{~K}) d_{\mathrm{In}}^{2}$ is always larger than $d_{\text {Out }}^{2}$ (at long times the two curves collapse since the history of the In and Out atoms becomes the same on average). This difference is maximum after $\approx 0.1 \mathrm{~ns}$ when $d_{\text {In }}^{2}$ is twice as important than $d_{\text {Out }}^{2}$. This indicates clearly that the $\mathrm{Na}$ atoms are more mobile inside the channels. This result is coherent with the findings of Horbach et al. [9] who state that the "sodium atoms move quickly between preferential sites" but has never been quantified directly so far. It is confirmed in the inset of FIG. 6 (a) by the difference between the MSD of the $\mathrm{Na}$ atoms that are moving only inside the channels and the MSD of the sodium atoms moving only outside the channels. At $T=2300 \mathrm{~K}$ (FIG. 6 (c)), we see that the difference between $d_{\text {In }}^{2}$ and $d_{\text {Out }}^{2}$ vanishes even though 
the channels still exist. Nevertheless as shown in FIG. 3 at that temperature the channels are not static anymore and therefore the distinction between In and Out has no clear meaning. At even higher temperatures (not shown), this is of course also true, and the two curves are superimposed. We can hence conclude that when the channels are formed and "static" (lowest temperatures), the sodium diffusivity is higher when the $\mathrm{Na}$ atoms are inside the channels.

In summary, with the use of classical molecular dynamics simulations on $\mathrm{Na}_{2} \mathrm{O}-4 \mathrm{SiO}_{2}$ systems we have analyzed in more detail the properties of the sodium trajectories inside the glassy matrix and especially the differences between the $\mathrm{Na}$ atoms inside the diffusion channels and those outside of these channels (the existence of these channels defined as the fraction of space mostly visited by the $\mathrm{Na}$ atoms has been shown in a previous study [5]). Firstly we have shown that the average potential energy of the $\mathrm{Na}$ atoms is the same whether they are In or Out. This is also true concerning their local environment except a slightly higher $\mathrm{Na}$ coordination number for the sodium atoms inside the channels. This is coherent with the fact that, in comparison with the rest of the system, the concentration of sodium atoms at low temperature $(\mathrm{T} \leq 3000 \mathrm{~K})$ is higher inside the channels. At high temperature the matrix can not be considered as frozen and therefore the channels do not exist anymore. We have defined a characteristic residence time inside the channels $\tau(T)$ that shows an Arrhenius dependence with an activation energy around $1.45 \mathrm{eV}$. This value is slightly higher than the characteristic energy of the other activated processes present in this system. Finally, we have shown that, at low temperature when the channels can be considered as frozen, the mobility of the sodiums inside the channels is larger than the one of the $\mathrm{Na}$ atoms in the rest of the system: in that sense one can really speak about channel diffusion.

In order to find the origin of this effect and in particular the role of the $\mathrm{SiO}_{2}$ matrix, one might change the vibrational characteristics of the matrix or those of the cation and see how these changes affect the sodium dynamics. These are investigations currently under way.

We thank W. Kob for interesting discussions. Part of the numerical calculations were performed at "Centre Informatique National de l'Enseignement Supérieur" in Montpellier, France.

[1] R. Dupree, D. Holland, P.W. McMillan and R.F. Pettifer, J. Non-Cryst. Sol. 68, 399 (1984); J. F. Emerson, P.E. Stallworth and P.J. Bray, J.Non-Cryst. Sol. 113, 253 (1989); H. Maekawa, T. Maekawa, K. Kawamura and T. Yokokawa, J. Non-Cryst. Sol. 127, 53 (1991); J. Kümmerlen, L. H. Merwin, A. Sebald and H. Keppler,
J. Phys. Chem. 96, 6405 (1992); N. Zotov, H. Keppler, A.C. Hannon and A.K. Soper, J. Non-Cryst. Sol. 202, 153 (1996); N. Zotov and H. Keppler, Phys. Chem. Minerals 26, 107 (1998); N. Zotov and H. Keppler, Phys. Chem. Minerals 25, 259 (1998); G. H. Wolf, D.J. Durben and P. F. Mc Millan, J. Chem. Phys. 93, 2280 (1990); S.N. Houde-Walter, J.M. Inman, A.J. Dent and G. N. Greaves, J. Phys. Chem. 97, 9330 (1993); G. N. Greaves and K.L. Ngai, Phys. Rev. B 52, 6358 (1995).

[2] A. A. Tesar and A. K. Varshneya, J. Chem. Phys. 87, 2986 (1987); C. Huang and A. N. Cormack, J. Chem. Phys. 93, 8180 (1990); C. Huang and A. N. Cormack, J. Chem. Phys. 95, 3634 (1991); H. Melman and S. H. Garofalini, J. Non-Cryst. Sol. 134, 107 (1991); N. Zotov, I. Ebbsjö, D.Timpel and H. Keppler, Phys. Rev B 60, 6383 (1999); S. Ispas, M. Benoit, P. Jund and R. Jullien, Phys. Rev. B 64, 214206 (2001).

[3] S.R. Elliott and P.A. Owens, Ber. Bunsenges. Phys. Chem. 95, 987 (1991); A. Bunde, M.D. Ingram and P. Maass, J. Non-Cryst. Solids 172-174, 1222 (1994); D. Knoedler, P. Pendzig and W. Dieterich, Solid State Ionics 86-88, 29 (1996); K.Y. Tsang and K.L. Ngai, Phys. Rev. E 56 R17 (1997); K. Funke and D. Wilmer, in Solid State Ionics V, ed. by G.-A. Nazri, C. Julien and A. Rougier, Mater. Res. Soc. Symp. Proc. Proceedings No. 548 (Materials Research Society, Pittsburg, 1999), p. 403; P. Maass, J. Non-Cryst. Solids 255, 35 (1999); T.B. Schroder and J.C. Dyre, Phys. Rev. Lett. 84, 310 (2000).

[4] B. Roling, C. Martiny and S. Bruckner, Phys. Rev. B 63, 214203 (2001).

[5] P. Jund, W. Kob and R. Jullien, Phys. Rev. B 64, 134303 (2001).

[6] G.N. Greaves, J. Non-Cryst. Solids 71, 203 (1985); G.N. Greaves, Miner. Mag. 64, 441 (2000), and references therein.

[7] C. Huang and A.N. Cormack, J. Chem. Phys. 93 (11), 8180 (1990); B. Vessal, G.N. Greaves, P.T. Marten, A.V. Chadwick, R. Mole and S. Houde-Walter, Nature 356, 504 (1992); G.N. Greaves and K.L. Ngai, Phys. Rev. B 52, 6358 (1995)

[8] A. Meyer, H. Schober and D.B. Dingwell (submitted to Phys. Rev. Lett.).

[9] J. Horbach, W. Kob and K. Binder, (cond-mat/0110325)

[10] J. Oviedo and J.F. Sanz, Phys. Rev. B 58, 9047 (1998).

[11] E. Sunyer, P. Jund, W. Kob and R. Jullien, J. Non-Cryst. Solids (to be published).

[12] N. Bansal and R. Doremus, Handbook of Glass Properties, Academic Press, London (1986).

[13] G.J. Kramer, A.J.M. de Man and R.A. van Santen, J. Am. Chem. Soc. 64, 6435 (1991).

[14] J. Horbach, W. Kob, K. Binder, Philos. Mag. B 79 (1999) 1981; J. Horbach, W. Kob, K. Binder, Chem. Geol. 174, 87 (2001). 
Figure 1

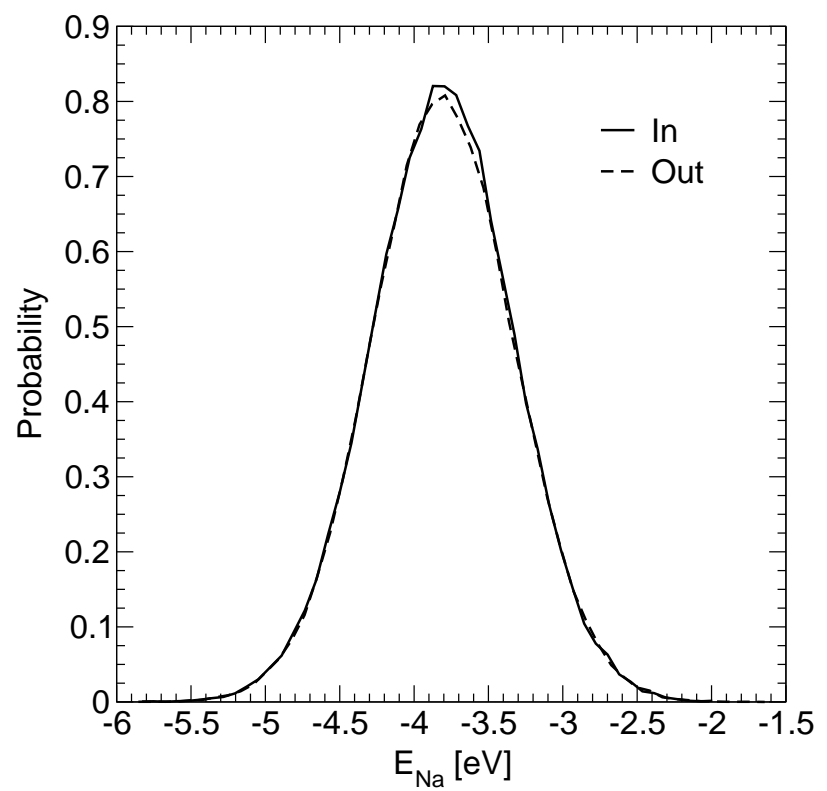

FIG. 1. Individual potential energy distributions of the sodium atoms inside and outside the channels (1700 K).

Figure 2

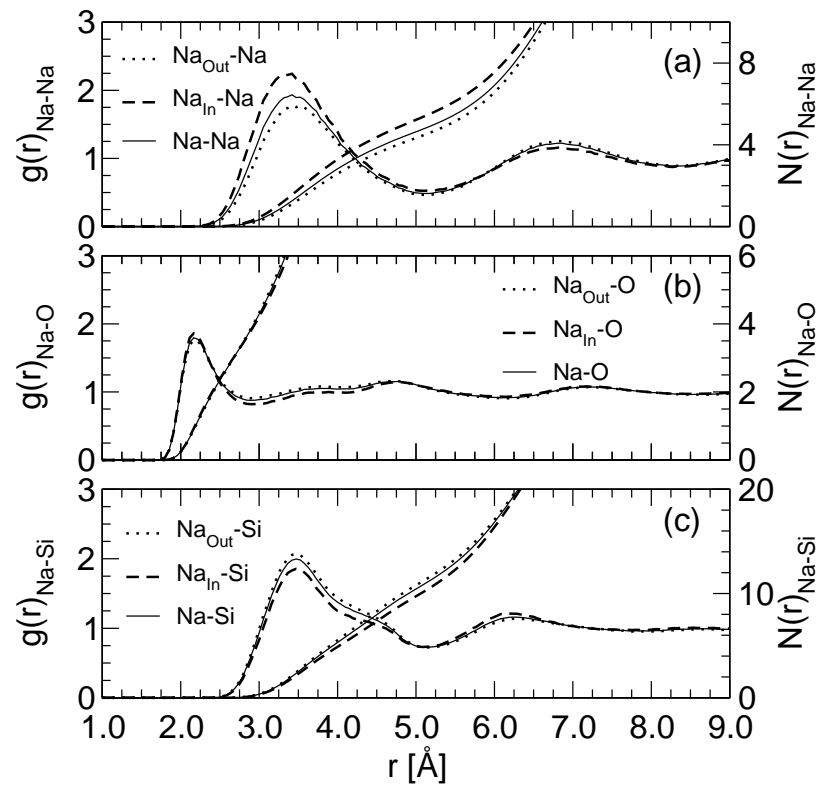

FIG. 2. Radial pair distribution functions, $g(r)$, and integrated numbers of neighbors, $N(r)$, at $1700 \mathrm{~K}$ for (a) NaOut $-\mathrm{Na}, \mathrm{Na}_{\mathrm{In}}-\mathrm{Na}$ and $\mathrm{Na}-\mathrm{Na}$ pairs, (b) NaOut $-\mathrm{O}$, $\mathrm{Na}_{\mathrm{In}}-\mathrm{O}$ and $\mathrm{Na}-\mathrm{O}$ pairs and (c) $\mathrm{NaOut}-\mathrm{Si}, \mathrm{Na}$ In $-\mathrm{Si}$ and $\mathrm{Na}-\mathrm{Si}$ pairs.
Figure 3

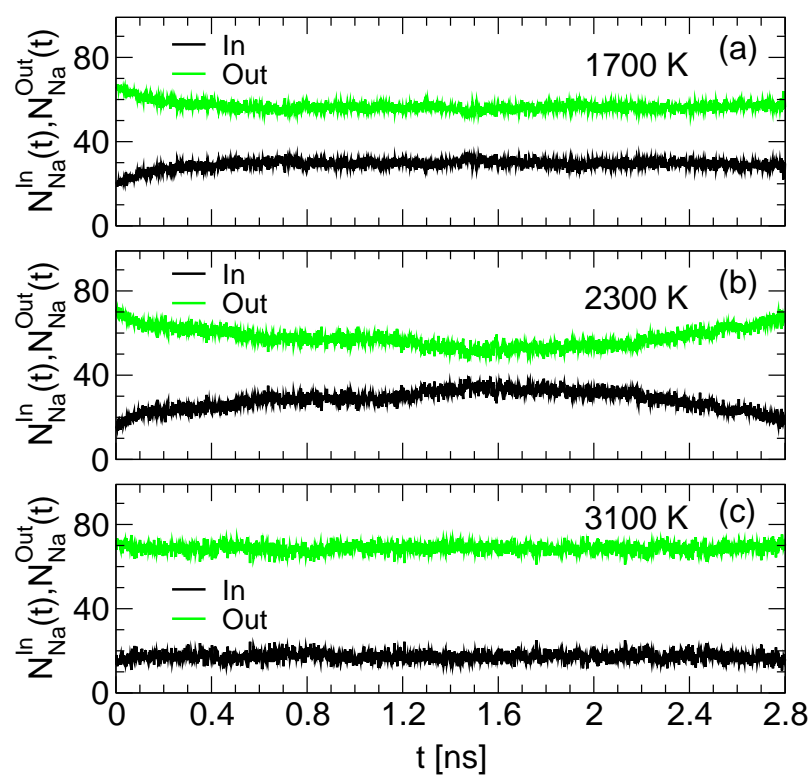

FIG. 3. Number of sodium atoms inside and outside the channels versus time at (a) $1700 \mathrm{~K}$, (b) $2300 \mathrm{~K}$ and (c) $3100 \mathrm{~K}$.

Figure 4

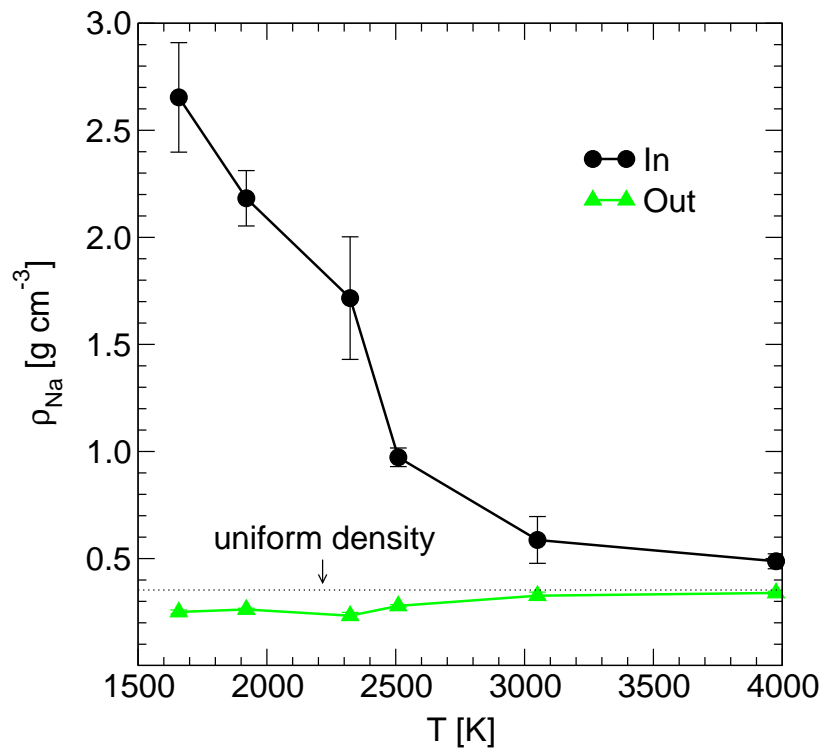

FIG. 4. Sodium density $\rho_{\mathrm{Na}}$ inside and outside the channels versus temperature. 
Figure 5

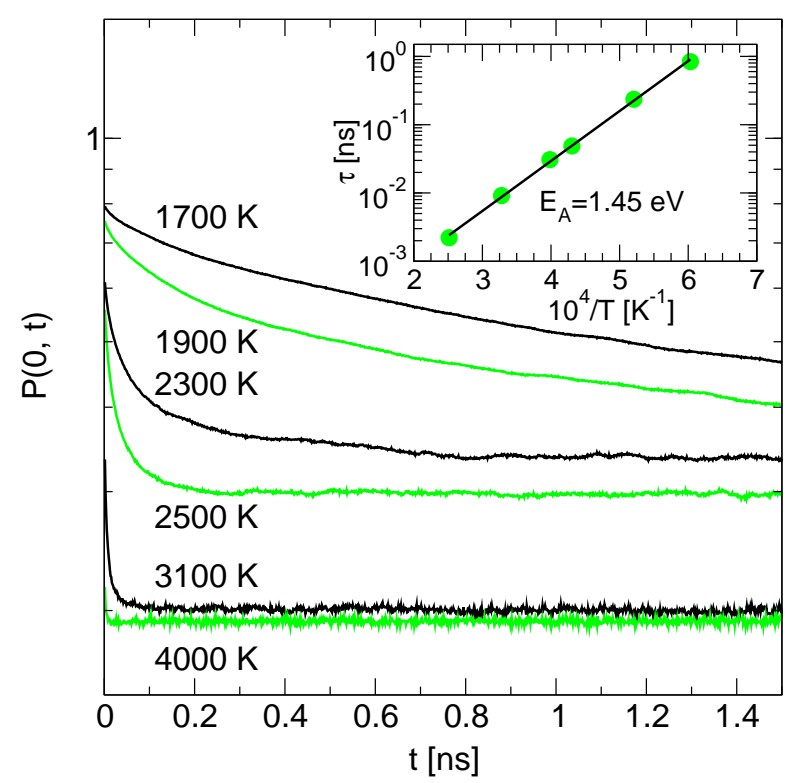

FIG. 5. Probability $P(0, t)$ that a sodium atom is inside the channels at time $t=0$ and time $t \neq 0$ for different temperatures. In inset: $\tau$ versus $1 / \mathrm{T}$ where $\tau$ is the time constant of an exponential fit of $P(0, t)$

Figure 6

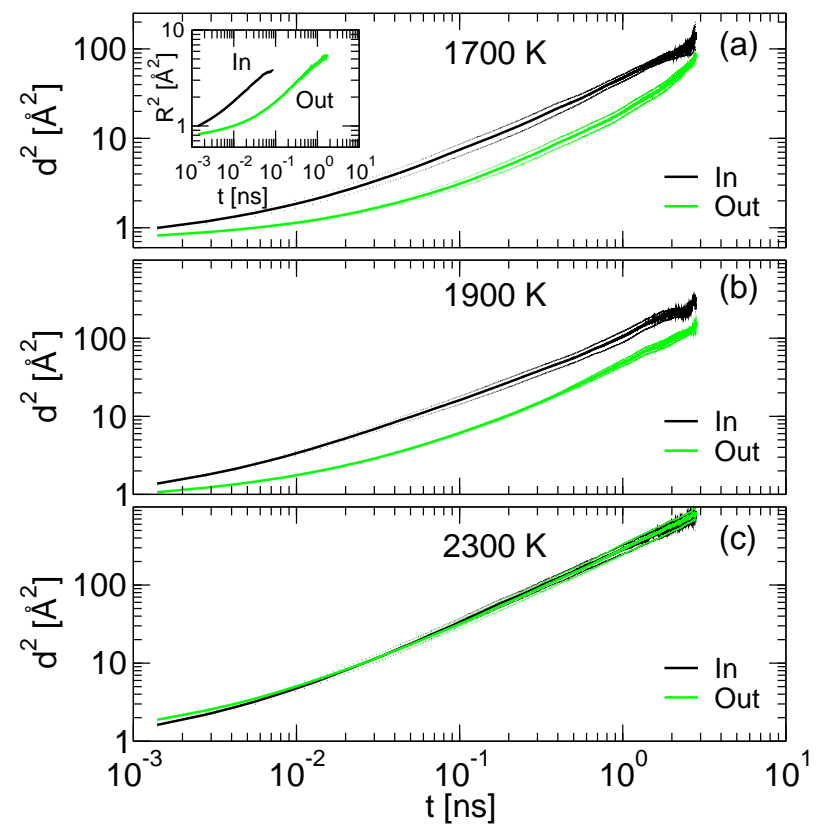

FIG. 6. $d^{2}(t)_{\text {In }}$ and $d^{2}(t)_{\text {Out }}$ (see text for definition) at (a) $1700 \mathrm{~K}$, (b) $1900 \mathrm{~K}$ and (c) $2300 \mathrm{~K}$. Inset: Mean square displacements $R^{2}(t)_{\text {In }}$ and $R^{2}(t)_{\text {Out }}$ (see text for definition) at $1700 \mathrm{~K}$. 